\title{
A New Method for Intelligent Check of Transformation Equipment Condition Monitoring Device
}

\author{
Hu Chengbo ${ }^{1, a}$, Ma Yong ${ }^{2, b}$, Zhou Zhicheng $^{3, \mathrm{c}}$, Tao Fengbo ${ }^{4, \mathrm{~d}}$, Xu Changfu $^{5, \mathrm{e}}$ \\ 1,2,3,4,5 Jiangsu Electric Power Company Research Institute, Nanjing, 211103, China \\ aemail:69972006@qq.com, bemail:my1313@163.com, email:zzc0708@163.com, \\ demail:t123456@163.com, eemail:xu523@163.com,
}

Keywords: Calibration; operation parameter; standard signal source; standard monitor equipment;

\begin{abstract}
Transformation equipment on-line monitoring device aims at monitoring substation equipment operation parameters. Through internal network, the data will be transported to the on-line monitoring platform of electric power internal network to provide maintenance basis for maintenance department. While the existing calibration method needs professional personnel carrying relevant calibrators and conducting contrast calibration on site. In order to make up for deficiency of existing substation on-line monitoring device calibration, such as instruments isolated with each other, instrument control and data management low automation, data contrast, retrieval and archiving is inconvenient, personnel requirement large and so on. This paper presents a new method for intelligent check of transformation equipment condition monitoring device based on wireless communication technology and computer control technology. It can promote the accuracy of calibration greatly and provide a powerful guarantee to grid reliable operation.
\end{abstract}

\section{Introduction}

Transformation equipment on-line monitoring device aims at monitoring substation equipment operation parameters. Through internal network, the data will be transported to the on-line monitoring platform of electric power internal network to provide maintenance basis for maintenance department. In order to guarantee the accuracy of monitoring data, it is very necessary to calibrate the monitoring device regularly. At present, calibration method needs professional personnel carrying relevant calibrators and conducting contrast calibration on site. This kind of replicate tests not only waste a large number of human resource, but also easy to cause artificial error. It will bring unsafe hidden danger to calibration work.

Aiming at the above problems, the paper presents a new method for intelligent check of transformation equipment condition monitoring device. This method is based on wireless communication technology and computer control technology, accesses the electric internal network through VPN wireless LAN environment and security accessing platform. Then obtain the real-time checked monitoring data from the on-line monitoring master station platform. Construct the wireless LAN environment that covered total station and create the stable and reliable encryption network link between kinds of standard signal source and standard monitoring devices. Realize the data calibration to common on-line monitoring devices by utilizing standard signal sources and standard monitoring devices.

\section{Status and Problems}

At present, the common process of calibration work as the following.

(1)According to the checked on-line monitoring device, professional personnel select the corresponding standard signal source and carry it to the site.

(2)After arriving the site, load the standard signal source to the checked on-line monitoring device. Regulate the output of standard signal source artificially and record the data.

(3)Login on electric internal network on-line monitoring master station platform, search for the real-time date during calibration and record them. 
(4)Repeat steps 2and 3, then obtain multi-group monitoring data which are in the full range.

(5)Contrast output signal data of standard signal source and the real-time monitoring data of the checked on-line monitoring device, draw the conclusion that whether the checked on-line monitoring data is correct.

Disadvantages of the existing calibration method is obvious.

(1)During the process of field calibration, the instruments are isolated with each other and there are not any data interaction.

(2)Standard signal source parameter and trigger action need to be regulated artificially. Replicate tests waste a large number of human resource and lead to low working.

(3)Acquisition of the checked monitoring date need to access the electric internal network on-line monitoring master station platform. This kind of artificial interface inquiry mode is inconvenient.

(4)The record and contrast of calibration data relay on artificial manipulation, it can easily cause artificial errors.

(5)Calibration results have not realize on-line maintenance. It is inconvenient to establish on-line monitoring device calibration record and afterwards retrieval.

(6)The development of calibration work needs experienced professional personnel. As the popular of transformation on-line monitoring device, the maintenance should be conducted regularly. It will cost a large number of human resources.

\section{Principle introduction}

In order to make up for deficiency of existing substation on-line monitoring device calibration, such as instruments isolated with each other, instrument control and data management low automation, data contrast 、 retrieval and archiving is inconvenient, personnel requirement large and so on. This paper presents a new method for intelligent check of transformation equipment condition monitoring device based on wireless communication technology and computer control technology.

Accesses the electric internal network through VPN wireless LAN environment and security accessing platform. Then obtain the real-time checked monitoring data from the on-line monitoring master station platform. Communicate with mobile audio and video monitoring terminal, standard signal source and handhold PDA through wireless LAN communication system. Design standard signal source output control scheme to realize real-time contrast standard signal source output data and checked on-line monitoring device monitoring data. Draw the conclusion through the corresponding software[1].

Utilize the handhold PDA to communicate with standard signal source through WIFI. The handhold PDA can operate the standard signal source after authorization.

Utilize the computer technology to develop human-computer interaction interface and realize presupposition and setting of standard signal source output scheme. Combined with database, record the checked on-line monitoring data and support query the historical records.

\section{Methods and steps}

The new method for intelligent check of transformation equipment condition monitoring device mainly includes the following steps.

(1)Drive the vehicle with communication center, standard signal source, wireless LAN communication system and mobile audio/video monitoring terminals to the checked substation.

(2)Open the corresponding software, query the ID of the checked on-line monitoring device and allocate it. If it is the first time to calibration, create archives. If the calibration archive is exist, query the record and prepare for the following calibration work.

(3)After construct the reliable link with electric internal network, reading the real-time data.

(4)Select the corresponding standard signal source and install it next to the checked on-line monitoring device. Load the output of standard signal source to the sensor of checked on-line 
monitoring device.

(5)Layout the wireless LAN network communication system transceiver end on the site and erect temporary wireless LAN network.

(6) Layout the mobile audio and video monitoring terminal on the site. Regulate audio signal and monitoring the calibration process.

(7)According to the historical calibration results, complicate calibration scheme. Send the scheme to the standard signal source. After the standard signal source returning results, the calibration work start-up.

(8)Contrast the standard signal source output data and the checked on-line monitoring data. According to the presupposition error range, judge the accuracy of the monitoring data.

(9)According to the situation, the handhold PDA can be authorized to operate the standard signal source output during the calibration. The standard signal source can be authorized to operate itself through operational key.

(10)When the calibration is over, generate the calibration report forms and store them[2-3].

\section{Standard Signal Source}

(1)Standard circuit breaker signal source

Circuit breaker standard signal source simulates the parameters of normal and abnormal state: primary circuit breaking current; switching closing coil current; storage energy motor energy; travel sensor voltage signal, current signal, digital signal[4]. The work flow as is shown in figure 1.

(2)Arrester\ capacitive equipmentliron core leakage current standard signal source.

Arrester, capacitive equipment, transformer iron core leakage current on-line monitoring device are all used to monitor the devices running states by observing the current, phase of above devices.

(3)Neutral point current standard signal source

Transformer neutral point current standard signal source adopts direct current standard source. The output voltage is $0 \sim 20 \mathrm{~V}$, output current amplitude is $0 \sim 50 \mathrm{~A}$, current precision is $0.1 \%$, output type is dc output. Transformer neutral point current standard signal source work diagram as is shown in figure 2.

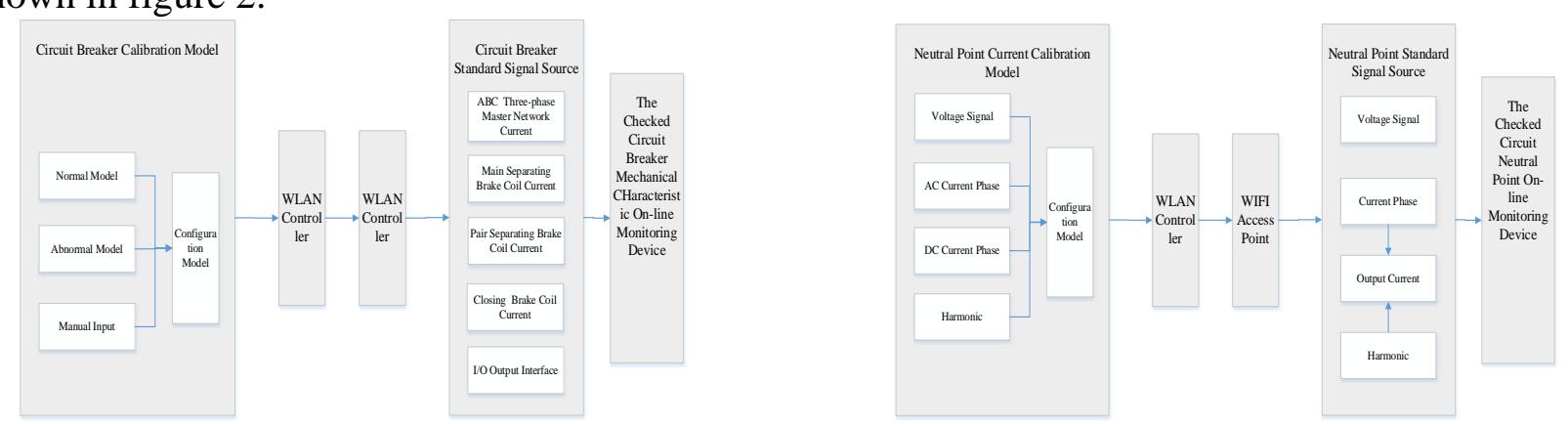

Fig 1Standard circuit breaker signal source Fig 2Transformer neutral point current standard signal source

(4)SF6 gas density standard signal source

SF6 gas density standard signal source adopts storage cylinder and variable cylinder. The standard signal source adopts diameter $250 \mathrm{~mm}$ tunable stroke cylinder as storage cylinder. Inner set pressure sensor and temperature and humidity sensor, regulate the internal SF6 gas pressure by electric piston to the expected pressure value and ratio to checked device.

\section{Standard Monitoring Device}

(1)Circuit breaker mechanical characteristic standard on-line device

Standard circuit breaker mechanical characteristic on-line monitoring device adopts synchronous sampling technique. Collect primary circuit breaking current, switching closing coil current, stored energy motor current parameter through current sensor. Collect contact signal through auxiliary contact. Move the device through stroke sensor collect contact. The collected signals can provide assistance to monitor circuit breaker mechanical characteristic state and evaluate electrical 
endurance through record analysis.

(2)Standard arrester/capacitive equipmentliron core current on-line monitoring device

Arrester/capacitive equipmentliron core current on-line monitoring device is composed of system power monitoring unit(IMM-U), capacitive equipment monitoring unit(IMM-C), field environment monitoring unit(IMM-E), iron core grounding current monitoring unit(IMM-I), MOA arrester monitoring unit(IMM-M), data treatment server(IMM-Z) and WIFI interface[5].

(3)Standard neutral point current on-line monitoring device

Standard neutral point current on-line monitoring device is aiming at observing the effect of HVDC to ac network transformer. It can also monitor transformer neutral point dc current, transformer noises and vibration.

(4)Standard SF6 gas density micro-water on-line monitoring device

Standard SF6 gas density micro-water on-line monitoring device is composed of sensor unit, data collector and data server. The high precision pressure, temperature, humidity transmitter of sensor unit are transformed to data signal by $\mathrm{A} / \mathrm{D}$ and conduct compensating operation by microprocessor. The collected data is send to SF6 micro-water monitoring unit, the indicator will display SF6 gas temperature, pressure, density, volume ratio and dew point of the monitored high pressure equipment[6].

(5)Standard SF6 micro-water high precision monitoring device

Standard SF6 micro-water high precision monitoring device adopts light dew indicator(Optidew Vision) which is a combination of the latest transmitter design and data control technique. The working principle is based on optical dew point measurement and it has the ability of test constantly, high flexibility and high precision.

\section{Conclusion}

This method solves the questions such as artificial operation, inconvenient archiving and retrieval. It meets the need of intelligent calibration of substation on-line monitoring devices and has a very wide market.

\section{References}

[1]SHEN Zhu, MEI Zhi-gang, ZHAO Wei, et al. calibrator for electronic current transformer[J]. J Tsinghua univ(sci\&tech), 2003, (3): 314-317.

[2]WU Gui-chu, ZEN Yun-liang, LI Han, et al. Design of Online Test System Of Circuit Breaker[J]. Bulletin of Science and Technology, 2007,23(6):873-877.

[3]CHEN Jiulin, XU Chencheng, WU Zaijun, DOU Xiaobo, et al. Development of Transmission Line On-line Monitoring System Based On Wireless Network[J]. Jiangsu Electrical Engineering, 2013, 32(6): 39-42

[4] REN Shi-chao, WANG Xian-zhong, CHEN Fu-qiang. Design of online sf6 instrument calibration device based on ARM[J]. Instrument Technique and Senseor, 2014,(12):39-41.

[5] CHEN Zhong-mei, WU Ying-jun, LIU Shuai, et al. Calibration Device of Arrester On-line Monitoring Based on High Precision Current Meter[J]. Industrial Control Computer, 2012,(5):120-122.

[6]SHU Jia, HUANG Xin-bo, ZHAO Long, et al. Design of SF6 Gas Intelligence Monitoring Device for Smart Substation[J]. High Voltage Engineering, 2013， 49(4):1-6. 